Rodrigues, F., Cid, L., Forte, P., Gonçalves, C., Machado, S., Neiva, H., Teixeira, D., \& Monteiro, D. (2020) The perception of enjoyment in youth, adults, and elderly: A Comparative study. Cuadernos de Psicología del Deporte, 20(2), 26-36

\title{
A perceção de divertimento em jovens, adultos e idosos: um estudo comparativo
}

\section{The perception of enjoyment in youth, adults, and elderly: a comparative study}

\section{La percepción del disfrute en jóvenes, adultos y ancianos: un estudio comparativo}

Rodrigues, F.1,2,3, Cid, L.1,2, Forte, P.2,4,5, Gonçalves, C.6,7, Machado, S.8,9,10, Neiva, H.2,11, Teixeira, D. 12, \& Monteiro, D. 1,2

I Sport Science School of Rio Maior (ESDRM - IPSantarém), Rio Maior, Portugal; 2 Research Center in Sport, Health Sciences and Human Development (CIDESD), Vila Real, Portugal; 3Research Center in Life Quality (CIEQV), Santarém, Portugal; 4 Instituto Superior de Ciências Educativas (ISCE Douro), Penafiel, Portugal; s Instituto Politécnico de Bragança, (IPB), Bragança, Portugal; 6 Faculdade de Medicina da Universidade do Porto (FMUP), Porto, Portugal; 7 Instituto de Ciências Biomédicas Abel Salazar (ICBAS), Porto, Portugal; sLaboratory of Physical Activity Neuroscience (LABNAF), Salgado de Oliveira University, Brazil,; 9 Laboratory of Panic and Respiration (LABPR), Institute of Psychiatry of Federal University of Rio de Janeiro (IPUB/UFRJ), Brazil; 1oIntercontinental Neuroscience Research Group, Brazil; 11 Beira Interior University (UBI), Covilhã, Portugal; 12 University of Lusófona (ULHT), Lisbon, Portugal

\section{RESUMEN}

Objetivo: Este estudo teve como objetivo analisar a perceção de divertimento em diferentes grupos etários, nomeadamente entre jovens, adultos e idosos. Acrescido, analisámos os efeitos diretos e indiretos na relação da motivação intrínseca, do divertimento e da intenção, de forma a compreender a validade preditiva do divertimento na intenção de continuar na prática de atividade física. Métodos: Participaram ao todo 425 indivíduos Portugueses praticantes de atividade física regular. Todos os participantes preencheram o questionário online tendo em consideração todas as variáveis em análise. Resultados: Os resultados da análise do modelo de medida evidenciam que este apresentou ser invariante entre as faixas etárias. Tal como teoricamente proposto, o divertimento demonstrou ser um preditor significativo da intenção e uma consequência de maiores níveis de motivação intrínseca. Conclusão: A escala utilizada na medição da perceção de divertimento demonstra ser um instrumento fiável, ao qual é equivalente tanto para jovens, como para adultos e idosos. O divertimento deve ser medido e considerado como variável chave na análise dos níveis de atividade física reportados por indivíduos em diferentes faixas etárias.

Palavras clave: divertimento; motivação intrínseca; intenção; atividade física; invariância. 


\title{
A perceção de divertimento em jovens, adultos e idoso
}

\begin{abstract}
Objective: This study aimed to analyze the perception of enjoyment in different age groups, namely among young people, adults and elderly. In addition, we analyzed the predictive validity of enjoyment as a predictor of intentions to continue physical activity and as a consequence of intrinsic motivation. Methods: A total of 425 Portuguese individuals practicing regular physical activity participated in this study. All participants completed the online questionnaire considering all the variables under analysis. Results: Results showed that the measurement model was invariant among the age groups. As theoretically proposed, enjoyment showed to be a significant predictor of intention and a consequence of higher levels of intrinsic motivation. Conclusion: The scale used to measure the perception of enjoyment proves to be a reliable instrument, which is equivalent for both young people, adults and elderly. Enjoyment should be measured and considered as a key variable in the analysis of physical activity levels reported by individuals in different age groups.
\end{abstract}

Keywords: enjoyment; intrinsic motivation; intentions; physical activity; measurement invariance.

\section{RESUMO}

Objetivo: Este estudio tuvo como objetivo analizar la percepción de diversión en diferentes grupos de edad, es decir, entre jóvenes, adultos y ancianos. Además, analizamos la validez predictiva de la diversión como un predictor de la intención de continuar la actividad física y como consecuencia de la motivación intrínseca. Métodos: Participaron un total de 425 portugueses que practicaban actividad física regular. Todos los participantes completaron el cuestionario en línea teniendo en cuenta todas las variables bajo análisis. Resultados: Los resultados del análisis del modelo de medición muestran que era invariante entre los grupos de edad. Como se propone teóricamente, se ha demostrado que la diversión es un predictor significativo de intención y una consecuencia de niveles más altos de motivación intrínseca. Conclusión: La escala utilizada para medir la percepción de la diversión demuestra ser un instrumento confiable, que es equivalente tanto para jóvenes, adultos y ancianos. La diversión debe medirse y considerarse como una variable clave en el análisis de los niveles de actividad física informados por individuos en diferentes grupos de edad.

Palavras chave: diversión; motivación intrínseca; intención actividad física; invariancia.

\section{INTRODUÇÃO}

O divertimento tem vindo a ser associado como uma variável chave na prática contínua de atividade física ao longo dos últimos anos (Jekauc, Voelkle, Wagner, Mewes, \& Woll, 2013). De facto, o divertimento parece ser um forte preditor para a manutenção da prática de exercício físico em adultos (Rodrigues, Teixeira, Cid, \& Monteiro, 2019a) bem como na prática desportiva em jovens (Gardner, Magee, \& Vella, 2017). No entanto, apenas recentemente é que se tem vindo a medir a perceção de divertimento na população idosa em contexto da atividade física (Mullen et al., 2011). Tanto quanto se sabe, existe falta de informação sobre a perceção de divertimento adjacente à realização do comportamento nos idosos. De facto, até à data, não se sabe se os níveis de prazer/divertimento adjacentes à prática de atividade física são díspares entre grupos etários (Rodrigues, Moutão, Teixeira, Cid, \& Monteiro, 2019). Nesse sentido, a medição do divertimento em idosos, e a posterior comparação, parece ser imprescindível (Mullen et al., 2011). Os resultados poderão ajudar técnicos de saúde e do exercício físico a estruturarem e planearem adequadamente sessões de atividade física, de acordo com os seus níveis de divertimento, promovendo uma maior intenção de se manterem na prática durante mais tempo (Mullen et al., 2011; Rodrigues et al., 2018; Rodrigues, Teixeira, Cid, \& Monteiro, 2019b).

\section{Divertimento e prática de atividade física}

O divertimento é a sensação de prazer e satisfação que um indivíduo sente quando realiza um comportamento que gosta. Relativamente à prática de atividade física, o divertimento tem vindo a ser associado como variável mediadora entre diversos fatores cognitivos e motivacionais na perceção de competência (Jekauc et al., 2013), bem como desempenhar um papel crucial na realização do comportamento a longo prazo (Dishman et al., 2005). De acordo com a Teoria da Autodeterminação (TAD; Ryan \& Deci, 2017) uma pessoa que regula o seu comportamento de 


\section{Rodrigues, Cid, Forte, Gonçalves, Machado, Neiva, Teixeira, Monteiro}

forma intrínseca (i.e., referente ao comportamento que é impulsionado por recompensas internas) está mais direcionado a experienciar resultados positivos. Por outras palavras, a motivação intrínseca indica que o comportamento surge de dentro do indivíduo, porque é naturalmente satisfatório para este, estando posicionado a uma maior sensação de emoções positivas, tais como o divertimento (Rodrigues et al., 2019b). Por sua vez, é expectável que, quando se realiza um comportamento de que se goste, haja maiores intenções de vir a realizar esse comportamento no futuro (Hagger \& Chatzisarantis, 2009). No caso da prática de atividade física, quanto maior for a experiência positiva da realização do comportamento (i.e., sensação de prazer e divertimento), maior será a probabilidade de repetir o comportamento no futuro (Mullen et al., 2011; Ryan \& Deci, 2017). Os benefícios da prática da atividade física orientam-se quer para o prazer, quer para os benefícios da saúde mental. Assim, a associação entre o divertimento e o prazer são fundamentais para a adesão à atividade física, resultando posteriormente num bem estar físico relacionado com a saúde (Rodrigues et al., 2019a).

Em estudos empíricos, a perceção de divertimento tem vindo a ser associada a maiores níveis de prática desportiva e atividade física fora do contexto escolar (Gardner et al., 2017; Moore et al., 2009). Acresce ainda que, em adultos inscritos em ginásios/health clubs que percecionam divertimento e prazer durante as atividades fitness têm maiores níveis de adesão ( Rodrigues et al., 2019a). No entanto, no que diz respeito à medição e perceção de divertimento em idosos, a literatura continua a ser escassa. Até à data, poucos estudos têm medido e considerado o divertimento como variável chave na prática de atividade física (Mullen et al., 2011). Neste sentido Mullen et al. (2011) referem que se devem realizar mais estudos nesta população, dado a relação entre o divertimento e a prática de atividade física, bem como os benefícios adjacentes à realização do comportamento serem essenciais no bem-estar e na longevidade desta população.

Apesar da crescente necessidade de medir o divertimento, como promotor de atividade física, até o momento, a capacidade de medir o divertimento em grupos de faixas etárias diferentes usando o mesmo instrumento permanece desconhecida. De facto, análises multi-grupos têm demostrado resultados contraditórios entre géneros (Moore et al., 2009). Além disso, não há evidências por sua validade fatorial e invariância entre as faixas etárias em populações que não sejam nativas das escalas de medição.
Este facto é ainda mais relevante em estudos que analisem o efeito preditor do divertimento na intenção de continuar na prática de atividade física, bem como a consequência da motivação intrínseca (Ryan \& Deci, 2017).

\section{Presente estudo}

Este estudo teve como objetivo de analisar a perceção de divertimento em diferentes grupos etários, nomeadamente entre jovens, adultos e idosos. Especificamente, pretendeu-se analisar as propriedades psicométricas do Physical Activity Enjoyment Scale (PACES)(Mullen et al., 2011), em grupos com faixas etárias diferentes (i.e., jovens, adultos e idosos). Foi ainda analisada a validade preditiva do divertimento na intenção de continuar na prática de atividade física, e como consequência da motivação intrínseca. De acordo com a literatura, especula-se que; a) o PACES será equivalente entre grupos com faixas etárias diferentes (Mullen et al., 2011); o divertimento é um preditor significativo da intenção de continuar na prática de atividade física (Gardner et al., 2017; Rodrigues et al., 2019a); e c) o divertimento é consequência da motivação intrínseca (Deci \& Ryan, 1985; Ryan \& Deci, 2017).

\section{MÉTODOS}

\section{Participantes}

No total, participaram neste estudo 425 indivíduos $($ sexo feminino $=231)$ praticantes de atividade física em contexto de ginásio e/ou health clubs em Portugal. Os praticantes tinham uma experiência de prática de atividade física regular entre 6 a 40 meses $(M=21,34 \pm S D=4,12)$ e uma prática semanal entre 2 a 5 vezes por semana $(M=2,43$ $\pm \mathrm{SD}=0,98) . \mathrm{A}$ atividade física praticada era primordialmente focada na componente de trabalho de força e capacidade cardiorrespiratória na sala de exercício (89\%), no entanto os participantes também realizavam regularmente aulas de grupo $(11 \%)$. Os participantes foram classificados como jovens ( $\leq 18$ anos), adultos ( $>18$ e $<65$ anos) e idosos ( $\geq 65$ anos) de acordo com a Organização Mundial de Saúde. Na Tabela 1 estão as características de cada grupo relativamente à média de idade, número de participantes e experiência da prática de atividade física. 


\section{A perceção de divertimento em jovens, adultos e idoso}

Tabela 1. Descrição sociodemográfica de cada amostra

\begin{tabular}{lccccccc}
\hline & $\mathrm{n}$ & $\mathrm{F}$ & $\mathrm{M}$ & Midade & DPidade & MExp & DPExp \\
\hline Jovens & 134 & 77 & 57 & 16.58 & .65 & 21.34 & 3.98 \\
Adultos & 166 & 105 & 61 & 36.16 & 3.21 & 25.12 & 2.66 \\
Idosos & 125 & 49 & 76 & 68.23 & .97 & 17.65 & 1.89
\end{tabular}

Notas: $\mathrm{n}=$ amostra; $\mathrm{F}=$ Feminino $; \mathrm{M}=$ Masculino; Midade $=$ Média de idade DPidade = Desvio-Padrão da idade; MExp = Média de Experiência; DPExp = Desvio-Padrão da Experiência.

\section{Instrumentos}

Para a recolha de dados relativamente à perceção do divertimento, utilizamos o Physical Activity Enjoyment Scale (PACES) traduzida e validada para a população Portuguesa (Teques et al., 2017). Esta escala é composta por oito itens (e.g., "É revigorante") precedida pela afirmação "A atividade física que pratico..." ao qual os participantes respondem através de uma escala tipo Likert com pontuação mínima 1 ("discordo totalmente") e máxima 7 (“concordo totalmente").

Como forma de medir a motivação intrínseca, usamos três itens do Behavioral Regulation in Exercise Questionnaire 3 (BREQ-3) traduzido e validado para a população portuguesa (Cid et al., 2018) no contexto da atividade física. Estes três itens (e.g., "Acho que é uma atividade agradável") avaliam especificamente a forma como a pessoa regula o seu comportamento de forma intrínseca. As opções de resposta são dadas através de uma escala tipo Likert com pontuação mínima 0 ("discordo totalmente") e máxima 4 ("concordo totalmente").

Para avaliar a intenção de se manter na prática de atividade física, foram criados três itens (e.g., "Pretendo manter a prática de atividade física no mesmo registo que pratico atualmente nos próximos meses") com base nas recomendações de Ajzen (2006), e com base no sistema Objetivo, Ação, Contexto e Tempo (Target, Action, Context, Time; ACT) proposto por este autor, ao qual os participantes responderam a cada item através de uma escala tipo Likert com pontuação mínima 1 ("discordo totalmente") e máxima 5 ("concordo totalmente").

\section{Procedimentos}

Estudo foi aprovado pela comissão ética (número de registo: CE-UBI-pJ-2018-044:ID683) previamente à recolha de dados. Acrescido, o processo de recolha dos dados esteve de acordo com a Declaração de Helsínquia e as alterações consequentes. Diversos gestores de ginásios e health clubs $(n=3)$ foram contactados por conveniência para a recolha de dados. Após explicação dos objetivos e aprovação por parte dos gestores, os investigadores contactaram presencialmente os potenciais participantes na zona de receção dos ginásios e health clubs. Foi explicado a todos os participantes os objetivos do estudo e foi-lhes providenciada a informação de anonimato. Os participantes preencheram o questionário através de uma plataforma online (i.e., surveymonkey). Todos os participantes assinaram consentimento informado previamente ao preenchimento do questionário. $\mathrm{O}$ tempo de preenchimento do questionário foi aproximadamente de 10 minutos.

\section{Análises Estatísticas}

Dado o questionário ter sido criado de forma a não existirem valores omissos, avançamos no cálculo de dados descritivos (média, desvio-padrão, assimetria e curtose) bem como de correlações bivariadas de Pearson com recurso ao programa estatístico IBM SPSS Statistics v23 (Arbuckle, 2013). Para aceitação de uma distribuição normal univariada, os valores de assimetria e de curtose devem estar contidos entre $-2 /+2$ e $-7 /+7$, respetivamente (Gravetter \& Wallnau, 2014).

\section{Estrutura Fatorial}

A estrutura fatorial do questionário foi avaliada através de uma Análise Fatorial Confirmatória (AFC) com recurso ao programa estatístico Mplus 7.4 (Muthén \& Muthén, 2010) e com o estimador Robust Maximum Likelihood (MLR) dado ser robusto contra a não normalidade da distribuição da amostra. Tomando em consideração que o teste do qui quadrado pode ser influenciado pelo tamanho amostral (Hair, Black, Babin, \& Anderson, 2019), consideramos os seguintes índices incrementais e absolutos para a aceitação do modelo de medida, dado não serem influenciados pelo tamanho da amostra e a complexidade do modelo (Hair et al., 2019; Sivo, Fan, Witta, \& Willse, 2006): Comparative Fit Index (CFI); Tucker-Lewis Index (TLI); Standardized Root Mean Residual (SRMR); Root Mean Square Error of Approximation (RMSEA) e o seu respetivo Intervalo de Confiança a 90\% (IC90\%). Para aceitação do modelo, foram considerados os seguintes valores de corte de acordo com as recomendações de diversos autores (Byrne, 2011; 


\section{Rodrigues, Cid, Forte, Gonçalves, Machado, Neiva, Teixeira, Monteiro}

Hair, Black, Babin, \& Anderson, 2014; Kline, 2016): CFI e TLI $\geq 0.90$, SRMR e RMSEA $\leq 0.80$.

De acordo com Hair et al. (2019), os pesos fatoriais devem ser $\geq 0.50$ de forma a que cada item explique pelo menos $25 \%$ de variância no fator latente. Acrescido, os coeficientes de fiabilidade compósita devem apresentar valores acima de 0.70 , tal como descrito por vários autores (e.g., Raykov, 1997). Foi também tido em consideração o cálculo da Variância Extraída Média (VEM), de forma a analisar a validade convergente dos itens. Para tal foi usado o valor de corte $\geq 0.50$ proposto por diversos autores (Hair et al., 2019; Kline, 2016).

\section{Análise Multi-grupos}

A análise de invariância do modelo de medida demonstra a capacidade de aplicabilidade do mesmo questionário em grupos com características diferentes (Sass, 2011). De facto, são vários os autores que descrevem a análise de invariância como uma análise fundamental na validação de escalas de medida (Chen, 2008; Cheung \& Rensvold, 2002). Nesse sentido, realizamos uma análise multi-grupos entre grupos etários (i.e., jovens, adultos e idosos) de acordo com recomendações de diversos autores (Byrne, 2011; Cheung \& Rensvold, 2002). Primeiramente, o modelo de medida deve ajustar-se em cada grupo (i.e., jovens, adultos e idosos). Seguidamente, foi testado a invariância configural (sem restrições de igualdade), invariância métrica (restrições nos pesos fatoriais dos itens), invariância escalar (restrições nos pesos fatoriais dos itens e nas interceções dos itens) e invariância residual (restrições nos pesos fatoriais dos itens, nas interceções e variância dos itens). As diferenças no CFI ( $\Delta \mathrm{CFI})$ e TLI ( $\Delta \mathrm{TLI})$ entre o modelo configural com o modelo métrico, escalar e residual deve ser inferior a 0.10 (Cheung \& Rensvold, 2002) e as diferenças no RMSEA ( $\triangle$ RMSEA) devem ser inferiores a 0.15 (Chen, 2007).

\section{Validade Preditiva}

A validade preditiva será analisada através de um modelo de equações estruturais (MEE) de acordo com a abordagem sugerida por Kline (2016). Primeiramente foi testado uma AFC tomando em consideração todas as variáveis em análise. No passo seguinte realizou-se um MEE tendo como base os pressupostos teóricos: a motivação intrínseca $\rightarrow$ divertimento $\rightarrow$ intenção. O ajustamento dos modelos resultantes da AFC e o modelo MEE serão analisados de acordo Hair et al. (2019), considerando os índices incrementais e absolutos tal como descritos anteriormente. Os coeficientes estandardizados serão considerados significativos caso o Intervalo de Confiança a 95\% (IC95\%) não englobe zero (Williams \& Mackinnon, 2008).

\section{RESULTADOS}

\section{Análise Preliminar}

De acordo com a Tabela 2, a perceção de divertimento foi a variável que apresentou a média mais alta comparativamente à motivação intrínseca e a intenção de continuar na prática de atividade física. Todas as variáveis apresentaram uma distribuição normal, dados os valores de assimetria e curtose estarem contidos dentro dos parâmetros aceitáveis. De acordo com os coeficientes de fiabilidade compósita, todas os grupos apresentaram valores acima do recomendado (jovens $=0.95$; adultos $=0.95$; idosos $=0.94$ ) Estrutura Fatorial

A AFC demonstra valores de ajustamento aceitáveis em todos os grupos em análise (ver Tabela 3). Especificamente, é possível de observar na Tabela 4 que os índices tradicionais e absolutos respeitam as recomendações, ao mesmo tempo que, os pesos fatoriais estão acima do valor de corte. De facto, é possível de observar que os pesos fatoriais do modelo de medida considerando a amostra total apresentam valores acima de 0.50 , explicando pelo menos $25 \%$ de variância no fator latente (ver Figura 1). Tomando em consideração os valores obtidos dos pesos fatoriais, é possível de observar que os valores da VEM são superiores aos valores de corte (jovens $=0.84$; adultos $=0.83$; idosos $=0.82$ ).

\section{Análise Multi-grupos}

De acordo com os resultados da análise multigrupos descritos na Tabela 5, é possível de afirmar que o modelo de medida da perceção do divertimento é invariante entre faixas etárias. De facto, a perceção parece ser equivalente entre grupos dados as diferenças no CFI e TLI serem inferiores a 0.10 .

\section{Validade Preditiva}

Foram encontrados efeitos diretos significativos tal como teoreticamente proposto: a motivação intrínseca apresentou ser um preditor positivo e significativo do divertimento; e o divertimento apresentou ser um preditor positivo e significativo da intenção em continuar na prática de atividade física divertimento. Acrescido, a motivação intrínseca apresentou um efeito indireto positivo e 


\section{A perceção de divertimento em jovens, adultos e idoso}

significativo na intenção continuar na prática de atividade física. Para mais informações ver Tabela 6.

Tabela 2. Análise descritiva, fiabilidade compósita e matriz de correlação

\begin{tabular}{lccccccc}
\hline & M & DP & A & C & \multicolumn{3}{c}{ Matriz de Correlação } \\
\cline { 6 - 8 } & & & & & 1 & 2 & 3 \\
\hline 1. Divertimento & 5.87 & .93 & -.51 & .10 & 1 & & \\
2. Motivação Intrínseca & 3.38 & .64 & -1.31 & 2.01 & $.69^{* *}$ & 1 & \\
3. Intenção & 4.54 & .67 & -1.90 & 1.84 & $.38^{* *}$ & $.31^{* *}$ & 1
\end{tabular}

Notas: $\mathrm{M}=$ Média; $\mathrm{DP}=$ Desvio-Padrão; $\mathrm{A}=$ Assimetria; $\mathrm{C}=$ Curtose $; * *<<0.01$

Tabela 3. Valores de ajustamento dos modelos

\begin{tabular}{lcccccc}
\hline Modelo & $\chi^{2}$ & gl & CFI & TLI & SRMR & RMSEA [90\% CI] \\
\hline 1. AFC amostra total & $160.134^{*}$ & 20 & .964 & .924 & .036 & $.064[.060, .068]$ \\
2. AFC jovens & $158.142^{*}$ & 20 & .954 & .919 & .040 & $.066[.059, .071]$ \\
3. AFC adultos & $170.787^{*}$ & 20 & .943 & .914 & .042 & $.063[.061, .066]$ \\
4. AFC idosos & $159.174^{*}$ & 20 & .967 & .929 & .032 & $.059[.054, .061]$ \\
5. MEE amostra total & $379.360^{*}$ & 87 & .921 & .905 & .036 & $.076[.069, .084]$ \\
6. MEE jovens & $435.321^{*}$ & 87 & .929 & .909 & .034 & $.068[.064, .072]$ \\
7. MEE adultos & $321.431^{*}$ & 87 & .931 & .913 & .032 & $.067[.062, .070]$ \\
8. MEE idosos & $578.147^{*}$ & 87 & .921 & .908 & .040 & $.072[.069, .078]$
\end{tabular}

Notas: $\chi^{2}=$ teste qui-quadrado; gl = graus de Liberdade; CFI = Comparative Fit Index; TLI = Tucker-Lewis Index; SRMR = Standardized Root Mean Square Residual; RMSEA $=$ Root Mean Squared Error of Approximation; CI90\% $=90 \%$ Intervalo de Confiança a $90 \%$ do RSMEA; $* p<0.001$

Tabela 4. Pesos fatoriais estandardizados em todos os grupos

\begin{tabular}{|c|c|c|c|c|c|c|c|c|c|}
\hline & \multirow{3}{*}{ Afirmação } & \multicolumn{3}{|c|}{ Jovens } & \multicolumn{3}{|c|}{ Adultos } & \multicolumn{2}{|c|}{ Idoso } \\
\hline & & \multirow{2}{*}{$\beta$ (SE) } & \multicolumn{2}{|c|}{ IC95\% } & \multirow[t]{2}{*}{$\beta$ (SE) } & \multicolumn{2}{|c|}{ IC95\% } & \multirow[t]{2}{*}{$\beta$ (SE) } & \multirow{2}{*}{$\frac{\text { IC9 }}{\text { LI }}$} \\
\hline & & & LI & LS & & LI & LS & & \\
\hline Item 1 & Acho que é agradável & $.81(02)$ & .76 & .84 & $.80(02)$ & .75 & .83 & $.80(02)$ & .74 \\
\hline Item 2 & É muito refrescante & $.93(01)$ & .92 & .95 & $.88(02)$ & .84 & .93 & $.91(01)$ & .88 \\
\hline Item 3 & É muito porreira & $.90(02)$ & .86 & .94 & $.93(01)$ & .85 & .94 & $.89(02)$ & .85 \\
\hline Item 4 & É muito revigorizante & $.70(05)$ & .60 & .77 & $.68(05)$ & .61 & .75 & $.70(05)$ & .62 \\
\hline Item 5 & É muito gratificante & $.82(02)$ & .75 & .85 & $.84(03)$ & .78 & .84 & $.79(03)$ & .74 \\
\hline Item 6 & $\dot{E}$ muito animada & $.90(02)$ & .86 & .93 & $.91(02)$ & .85 & .95 & $.89(02)$ & .87 \\
\hline Item 7 & É muito estimulante & $.89(02)$ & .85 & .90 & $.88(02)$ & .84 & .91 & $.88(02)$ & .83 \\
\hline Item 8 & É muito divertida & $.74(04)$ & .65 & .76 & $.68(03)$ & .64 & .77 & $.70(03)$ & .67 \\
\hline
\end{tabular}

Notas: Os coeficientes e erros da estimativa são apresentados com valores estandardizados; valores $\epsilon$ parênteses (erro da estimativa); Intervalo de Confiança a 95\%; todos os pesos fatoriais são significativos. 


\section{Rodrigues, Cid, Forte, Gonçalves, Machado, Neiva, Teixeira, Monteiro}

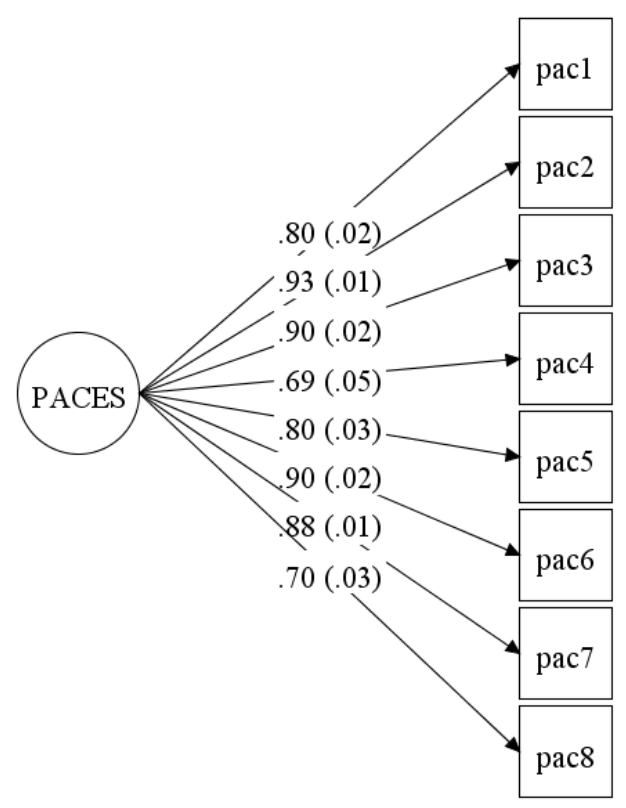

Figura 1. Modelo de medida do Physical Activity Enjoyment Scale com a amostra total

Notas: Os coeficientes e erros da estimativa são apresentados com valores estandardizados; valores entre parênteses (erro da estimativa); todos os pesos fatoriais são significativos.

Tabela 5. Análise multi-grupos do modelo de medida entre grupos etários

\begin{tabular}{lcccccc}
\hline Modelo & CFI & $\Delta$ CFI & TLI & $\Delta$ TLI & RMSEA & $\Delta$ RMSEA \\
\hline Invariância Configural & .961 & - & .969 & - & .077 & - \\
Invariância Métrica & .958 & .003 & .967 & .002 & .075 & .002 \\
Invariância Escalar & .958 & .003 & .967 & .002 & .074 & .003 \\
Invariância Residual & .960 & .001 & .969 & .000 & .070 & .007
\end{tabular}

Notas: CFI = Comparative Fit Index; $\triangle$ CFI = diferenças no CFI; TLI = Tucker-Lewis Index; $\triangle \mathrm{TLI}=$ diferenças no TLI; RMSEA $=$ RMSEA $=$ Root Mean Squared Error of Approximation; $\triangle \mathrm{RMSEA}=$ diferenças no RMSEA.

Tabela 6. Efeitos diretos e indiretos

\begin{tabular}{|c|c|c|c|c|c|c|c|c|}
\hline \multicolumn{3}{|c|}{ Jovens } & \multicolumn{3}{|c|}{ Adultos } & \multicolumn{3}{|c|}{ Idosos } \\
\hline \multirow{2}{*}{$\beta$} & \multicolumn{2}{|c|}{ IC95\% } & 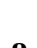 & \multicolumn{2}{|c|}{ IC95\% } & 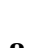 & \multicolumn{2}{|c|}{ IC95\% } \\
\hline & LI & LS & & LI & LS & & LI & LS \\
\hline
\end{tabular}

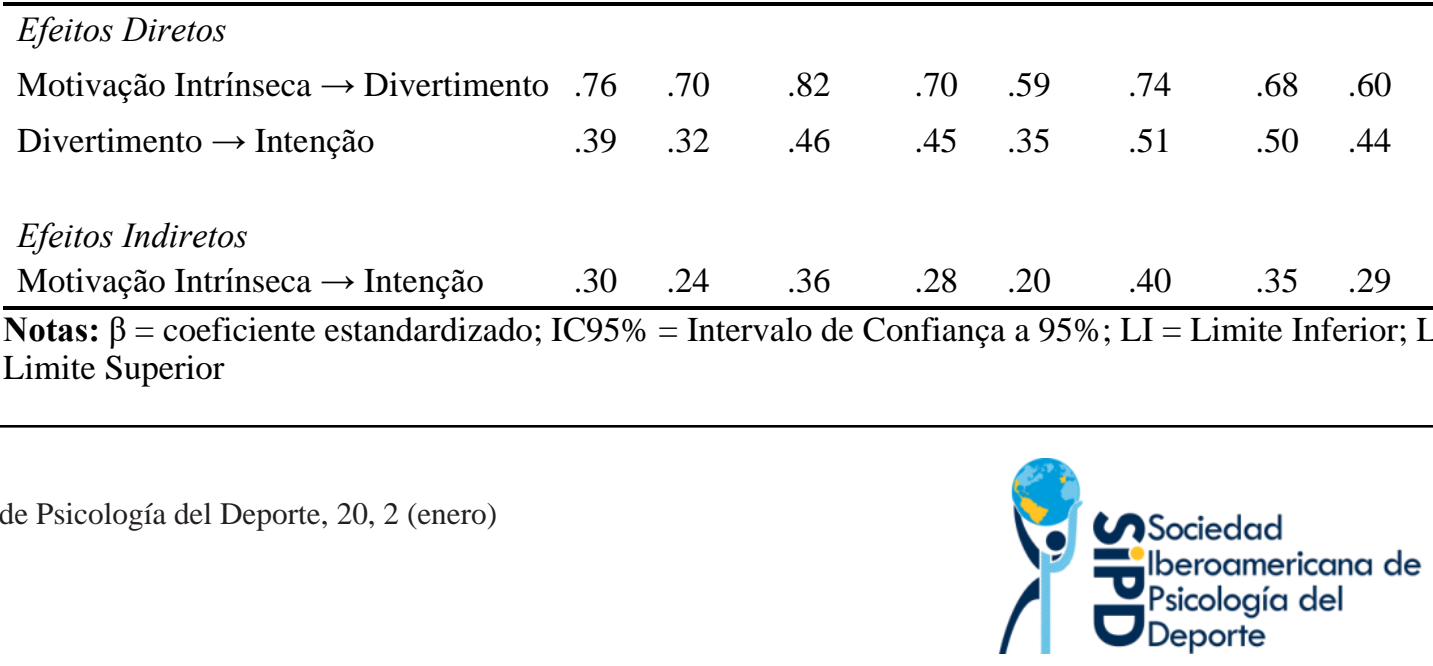




\section{A perceção de divertimento em jovens, adultos e idoso}

\section{DISCUSSÃO}

Este estudo teve como objetivo de analisar a perceção de divertimento em diferentes grupos etários, nomeadamente entre jovens, adultos e idosos. Especificamente, pretendeu-se comprovar a aplicabilidade e invariância do PACES numa amostra portuguesa praticante de atividade física em ginásios/health clubs. Qualquer diferença encontrada entre grupos implicaria variabilidade inerente à medição. No entanto, consistente com os resultados obtidos por Mullen et al. (2011), a versão de oito itens do PACES mostrou valores fiáveis nos diversos grupos.

\section{Estrutura Fatorial}

O primeiro passo consistiu na análise do modelo de medida nos diversos grupos. De acordo com os resultados, o modelo teve um ajustamento aceitável em todos os grupos, evidenciando propriedades psicométricas aceitáveis tal como proposto por vários autores (Byrne, 2011; Hair et al., 2019). Estes resultados são expectáveis, dado outros estudos terem aplicado a mesma escala em jovens (Motl et al., 2012), em adultos (Teques, Calmeiro, Silva, \& Borrego, 2017) e em idosos (Mullen et al., 2011). Os resultados também confirmaram consistência interna, na medida em que os valores da fiabilidade compósita são superiores aos valores de corte propostos por Hair et al. (2019). Nesse sentido, podemos afirmar que os oito itens medem o que é suposto medir, neste caso em concreto, a perceção de divertimento durante a prática de atividade física.

Olhando especificamente para os pesos fatoriais, foi possível observar que estes são elevados, sendo suportados pela literatura (Byrne, 2011; Kline, 2016), explicando mais que $25 \%$ de variância no fator latente.

\section{Análise Multi-grupos}

De acordo com os resultados, foi possível comprovar que o modelo de medida do PACES foi invariante entre faixas etárias, dado ter respeitado os critérios de invariância previamente descritos (Chen, 2007; Cheung \& Rensvold, 2002). Isto é, jovens, adultos e idosos percecionam de forma equivalente o divertimento durante a prática de atividade física.

\section{Validade Preditiva}

Tal como foi expectável, a perceção de divertimento esteve positivamente correlacionada com a motivação intrínseca e a intenção de continuar a na prática de atividade física. A validade preditiva foi demonstrada pelos efeitos positivos significativos entre a motivação intrínseca, divertimento e a intenção de continuar na prática. Estes resultados corroboram com estudos prévios (Monteiro et al., 2017; Rodrigues et al., 2019b) mostrando que um indivíduo que regula o seu comportamento de forma intrínseca está mais suscetível de experienciar respostas emocionais positivas, tais como o divertimento. Por sua vez, maiores níveis de divertimento estão subjacentes a uma maior intenção de continuar a realizar o comportamento no futuro. Estes resultados reforçam o papel chave do divertimento no que diz respeito à prática de atividade física, uma vez que a manutenção do comportamento está associada a um estilo de vida saudável (Hickey \& Mason, 2017), a uma qualidade de vida superior em pacientes oncológicos (Ungar, Wiskemann, \& Sieverding, 2016) e menor risco de contrair doenças crónicas não mutáveis (Andersen, Mota, \& Di Pietro, 2016).

Interessantemente, o valor preditivo da motivação intrínseca para o divertimento variou entre grupos, mostrando que grupos de pessoas com idades diferentes podem percecionar de forma diferente a sensação de prazer e divertimento adjacente à prática de exercício físico (Mullen et al., 2011). De acordo com os resultados obtidos, a motivação intrínseca tem um efeito maior para o divertimento nos jovens. Isto poderá estar relacionado com a vertente lúdica envolvida na atividade. Os jovens parecem estar envolvidos na prática de exercício físico por considerarem o comportamento algo prazeroso e que lhe promove uma sensação de divertimento. De facto, estudos anteriores têm demonstrado que jovens de ambos os sexos apresentam valores elevados de divertimento, seja em contexto de educação física (Dishman et al., 2005), bem como no contexto desportivo (Monteiro et al., 2017). Não obstante, o divertimento apresentou um efeito direto para a intenção maior nos idosos, comparativamente aos jovens e aos adultos. Igualmente, a motivação intrínseca apresentou um efeito indireto maior nos idosos comparativamente aos outros dois grupos praticantes de exercício físico. Estes resultados parecem corroborar com estudos prévios (Dacey, Baltzell, \& Zaichkowsky, 2008) evidenciando que os idosos que regulem o seu comportamento para a prática de exercício físico de forma intrínseca, considerando o comportamento como parte integrante deles, estão mais suscetíveis a sentirem prazer e divertimento durante a prática de exercício físico. Consequentemente, quando um sujeito realiza um comportamento do qual gosta e sente benefícios psicológicos adjacentes, apresenta maior 


\section{Rodrigues, Cid, Forte, Gonçalves, Machado, Neiva, Teixeira, Monteiro}

intenção de voltar a realizar e manter esse comportamento como parte integrante no quotidiano (Mullen et al., 2011).

\section{Limitações e agenda para estudos futuros}

Em primeiro lugar, este estudo foi realizado tendo em consideração uma amostra portuguesa praticante de atividade física, sendo por isso, os resultados deste estudo, limitados à população em questão. Assim, sugere-se a realização de mais estudos, especificamente de estudos interculturais, de forma a analisar a perceção do divertimento em diferentes grupos com faixas etárias diferentes. Em segundo lugar, este estudo é transversal no seu desenho metodológico. Nesse sentido, não é possível de evidenciar causalidade. Futuras investigações deverão realizar intervenções controladas de forma a estimular e promover maiores níveis de divertimento.

\section{CONCLUSÃO}

O PACES parece ser um instrumento fiável que avalia a perceção divertimento durante a prática de atividade física tanto em jovens, como em adultos e em idosos. Esta escala reduzida de oito itens é fiável na medição da perceção de divertimento em qualquer faixa etária analisada na população portuguesa, sendo também de rápida aplicação. De acordo com os resultados deste estudo, podemos afirmar que o PACES pode ser aplicado na medição da perceção divertimento em contexto da atividade física, como forma de avaliar o estado atual do praticante e como isso terá implicações na prática futura do comportamento.

\section{IMPLICAÇÕES PRÁCTICAS}

Em suma, os resultados poderão ajudar técnicos de saúde e de exercício físico a estruturarem e planearem adequadamente sessões de atividade física, de acordo com os seus níveis de divertimento. De facto, o acompanhamento personalizado e especializado, olhando para o ser humano como um ser emocional e não como um "objeto comercial", pode tornar a prática de atividade física como um comportamento prazeroso, resultando em maiores intenções e níveis de prática por parte da sociedade.

\section{REFERÊNCIAS}

Ajzen, I. (2006). Constructing a TPB questionnaire: Conceptual and methodological considerations.
Retrieved March 14, 2019, from http://wwwunix.oit.umass.edu/ aizen

Andersen, L., Mota, J., \& Di Pietro, L. (2016). Update on the global pandemic of physical inactivity. The Lancet, 388(10051), 1255-1256. doi: 10.1016/s0140-6736(16)30960-6

Arbuckle, J. (2013). IBM® SPSS ${ }^{\circledR}$ Amos $^{\mathrm{TM}} 22$ User's Guide: IBM Corp.

Byrne, B. (2011). Structural Equation Modeling with Mplus - Basic Concepts, Applications, and Programming (2nd ed.). Routledge. New York, USA.

Chen, F. (2007). Sensitivity of Goodness of Fit Indexes to lack of measurement invariance. Structural Equation Modeling: A Multidisciplinary Journal, 14(3), 464-504. doi: $10.1080 / 10705510701301834$

Chen, F. (2008). What happens if we compare chopsticks with forks? The impact of making inappropriate comparisons in cross-cultural research. Journal of Personality \& Social Psycholgy, 95(5), 1005-1018. doi: $10.1037 / \mathrm{a} 0013193$

Cheung, G., \& Rensvold, R. (2002). Evaluating Goodnessof-Fit Indexes for testing measurement invariance. Structural Equation Modeling: A Multidisciplinary Journal, 9(2), 233-255. doi: 10.1207/s15328007sem0902_5

Dacey, M., Baltzell, A., \& Zaichkowsky, L. (2008). Older adults' intrinsic and extrinsic motivation toward physical activity. American Journal of Health Behavior, 32(6), 570-582. doi: 10.5555/ajhb.2008.32.6.570

Deci, E., \& Ryan, R. (1985). Intrinsic Motivation and SelfDetermination in Human Behavior. Springer, Boston, USA.

Dishman, R. K., Motl, R. W., Saunders, R., Felton, G., Ward, D. S., Dowda, M., \& Pate, R. R. (2005). Enjoyment Mediates Effects of a School-Based Physical-Activity Intervention. Medicine \& Science in Sports \& Exercise, 37(3), 478-487. doi: 10.1249/01.mss.0000155391.62733.a7

Gardner, L., Magee, C., \& Vella, S. (2017). Enjoyment and Behavioral Intention Predict Organized Youth Sport Participation and Dropout. Journal of Physical Activity and Health, 14(11), 861-865. doi: 10.1123/jpah.2016-0572

Gravetter, F., \& Wallnau, L. (2014). Essentials of Statistics for the Behavioral Sciences (8th ed.). Cengage Learning. Wadsworth, Belmont, CA. 


\section{A perceção de divertimento em jovens, adultos e idoso}

Hair, J., Black, W., Babin, B., \& Anderson, R. (2014). Multivariate Data Analysis (7th ed.): New Jersey: Pearson Educational, Inc.

Hair, J., Black, W., Babin, B., \& Anderson, R. (2019). Multivariate Data Analysis (8th ed.): New Jersey: Pearson Educational, Inc.

Hickey, M., \& Mason, S. (2017). Age and gender differences in particpation rates, motivators for, and barriers to exercise. Modern Psychological Studies, 22(2), 10-19.

Jekauc, D., Voelkle, M., Wagner, M. O., Mewes, N., \& Woll, A. (2013). Reliability, validity, and measurement invariance of the German version of the physical activity enjoyment scale. Journal of Pediatric Psychology, 38(1), 104-115. doi: 10.1093/jpepsy/jss088

Kline, R. (2016). Principles and practice of structural equation modelling (3rd ed.): New York: The Guildford Press. USA.

Monteiro, D., Nunes, G., Marinho, D., Couto, N., Antunes, R., Moutão, J., \& Cid, L. (2017). Translation and adaptation of the physical activity enjoyment scale (PACES) in a sample of Portuguese athletes, invariance across genders nature sports and swimming. Brazilian Journal of Kinanthropometry and Human Performance, 19(6), 631-643. doi: 10.5007/1980-0037.2017v19n6p631

Moore, J., Yin, Z., Hanes, J., Duda, J., Gutin, B., \& Barbeau, P. (2009). Measuring Enjoyment of Physical Activity in Children: Validation of the Physical Activity Enjoyment Scale. Journal of Applied Sport Psychology, 21(S1), S116-S129. doi: 10.1080/10413200802593612

Motl, R. W., Dishman, R., Saunders, R., Dowda, M., Felton, G., \& Pate, R. R. (2012). Measuring enjoyment of physical activity in adolescent girls. American Journal of Preventive Medicine, 21(2), 110-117. doi: 10.1016/S0749-3797(01)00326-9

Mullen, S., Olson, E., Phillips, S., DSzabo, A., Wójcicki, T., Mailey, E., . . . McAuley, E. (2011). Measuring enjoyment of physical activity in older adults: invariance of the physical activity enjoyment scale (paces) across groups and time. International Journal of Behavioral Nutrition and Physical Activity, 8, 103. doi: 10.1186/1479-5868-8-103

Muthén, L., \& Muthén, B. (2010). Mplus User's Guide: Muthén \& Muthén: Los Angeles.
Raykov, T. (1997). Estimation of Composite Reliability for congeneric measures. Applied Psychological Measurement, 21(2), 173-184. doi: 10.1177/01466216970212006

Rodrigues, F., Bento, T., Cid, L., Pereira Neiva, H., Teixeira, D., Moutao, J., . . . Monteiro, D. (2018). Can Interpersonal Behavior Influence the Persistence and Adherence to Physical Exercise Practice in Adults? A Systematic Review. Frontiers in Psychology, 9, 2141. doi: 10.3389/fpsyg.2018.02141

Rodrigues, F., Teixeira, D., Cid, L., \& Monteiro, D. (2019a). Have you been exercising lately? Testing the role of past behavior on exercise adherence. Journal of Health Psychology, 1359105319878243 . doi: $10.1177 / 1359105319878243$

Rodrigues, F., Teixeira, D., Cid, L., \& Monteiro, D. (2019). Promoting exercise participation: the role of interpersonal behaviors for practical implications. Journal of Functional Morphology and Kinesiology, 4(2), 40. Doi: 10.3390/jfmk4020040

Ryan, R., \& Deci, E. (2017). Self-determination theory. Basic Psychological Needs in Motivation, Development, and Wellness: New York: Guildford Press. New York, USA.

Sass, D. (2011). Testing measurement invariance and comparing latent factor means within a Confirmatory Factor Analysis framework. Journal of Psychoeducational Assessment, 29(4), 347-363. doi: 10.1177/0734282911406661

Sivo, S. A., Fan, X., Witta, E. L., \& Willse, J. T. (2006). The Search for "Optimal" Cutoff Properties: Fit Index Criteria in Structural Equation Modeling. The Journal of Experimental Education, 74(3), 267-288. doi: 10.3200/jexe.74.3.267-288

Teques, P., Calmeiro, L., Silva, C., \& Borrego, C. (2017). Validation and adaptation of the Physical Activity Enjoyment Scale (PACES) in fitness group exercisers. Journal of Sport and Health Science. doi: 10.1016/j.jshs.2017.09.010

Ungar, N., Wiskemann, J., \& Sieverding, M. (2016). Physical Activity Enjoyment and Self-Efficacy As Predictors of Cancer Patients' Physical Activity Level. Frontiers in Psychology, 7, 898. doi: 10.3389/fpsyg.2016.00898

Williams, J., \& Mackinnon, D. (2008). Resampling and Distribution of the Product Methods for Testing 


\section{Rodrigues, Cid, Forte, Gonçalves, Machado, Neiva, Teixeira, Monteiro}

Indirect Effects in Complex Models. Structural Equation Modeling, 15(1), 23-51. doi: $10.1080 / 10705510701758166$

\section{Agradecimentos}

Este estudo foi suportado pela Fundação para Ciência e Tecnologia com o número de registo UID/DTP/04045/2019 para os autores LC, HN e DM. Este estudo também foi suportado pela Fundação para a Ciência e a Tecnologia, I.P., Projeto No UIDP/04748/2020 para o autor FR.

\section{Conflitos de interesse}

Nada a declarar. 